Journal of Engineering and Applied Sciences 14 (15): 4990-4995, 2019

ISSN: 1816-949X

(C) Medwell Journals, 2019

\title{
Video Mosaic Stylization using Traditional Mosaic Patterns
}

\author{
Jong-Chul Yoon \\ Division of Computer, Department of Software and Media Engineering, \\ Kangwon National University, Chuncheon, Korea media19@kangwon.ac.kr, +82-33-570-6869
}

\begin{abstract}
We present a novel mosaic stylization method for video which can conserve both temporal and spatial coherence. Based on three traditional mosaic patterns that were commonly used by ancient artists, our system assigns a selected mosaic pattern to segmented regions which conserve traditional mosaic design mechanisms and also effectively preserve spatial information. To preserve temporal coherence, we use update-based tiling approach that minimizes the flickering effect between frames. Using our system, non-professional users can easily generate a series of mosaic images with their own video clips.
\end{abstract}

Key words: Non-photorealistic rendering, mosaic stylization, video stylization, traditional mosaic pattern, generate, mechanisms

\section{INTRODUCTION}

In the computer graphics area, Non-Photorealistic Rendering (NPR) generally involves various stylizations of a scene such as abstraction, oil painting, line-drawing, and mosaic. The main goals of NPR are effectively emphasizing visual cues of a scene or mimic a traditional artistic presentation. Mosaic stylization is one example of artistic presentation and numerous techniques have been introduced (Dobashi et al., 2002; Mould, 2007; Elber and Wolberg, 2003; Kim and Pellacini, 2002).

To generate visually pleasing mosaics, researchers have tried to find a space-partitioning algorithm to determine a suitable size, position and angle of mosaic tiles. The Voronoi-diagram and blue noise sampling are well-known solutions to this problem (Dobashi et al., 2002; Mould, 2007). However, the algorithmic approach limited, since, it generates artificial mosaic patterns without considering the traditional patterns of man-made mosaics.

Moreover, the previous work on mosaic stylization only considered a single image as an input and did not extend to video because the temporal coherence of the tiles between frames is a bigger challenge in video mosaics. A conventional solution is to extract the motion vector fields along the time domain and then move the tiles along these vector fields. However, mosaic tiles generally have a squared shape (not pixels) and this causes significant flickering between frames due to changes in the tile angle and position.

In this study, we present a novel mosaic derived from traditional mosaic art with extendibility to video input. We first analyze traditional mosaic arts and extract three dominant tiling patterns. Then, we segment the input image into three different regions using spatial and directional complexity. After segmentation, we select the appropriate mosaic pattern for each region and place the mosaic tiles. To extend our framework to video input, we aligned video frames and calculate the differences to find the temporally changed regions. Then, we only update the tile information in the temporally changed region according to a feature of the image in the current frame while the other tiles maintain their information. In comparison to previous stylization approaches that move all the tiles along motion vectors, we only changed tiles in the temporally changed region to decrease the flicking effect between frames. The contribution of our paper as follows:

- Introduce enhanced mosaic stylization which follows a traditional man-made mosaic art

- Introduce a temporally coherent mosaic technique for video input

\section{MATERIALS AND METHODS}

Observation of traditional mosaic arts: Mosaic is the art of creating an image with small pieces of colored tiles. It has been used for decorative art and interior decorations from around $1500 \mathrm{BC}$. Historically several methods and materials are used for mosaic arts but three dominant tiling patterns have been commonly used. These three patterns are classified by variance of size, angle and shape of tiles.

Tessellatum is a tiling method that uses fixed tile shapes (generally squares) with different colors. It fills the region with tiles of a fixed size and angle, so, variations of tile size, angle and shape are not permitted. Figure la 
(a)

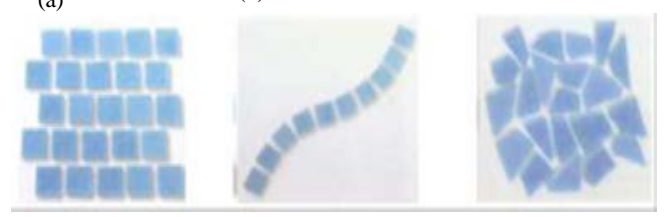

Fig. 1: Traditional mosaic patterns: a) Tessellatum pattern; b) Vermiculatum pattern and c) Palladianum pattern

shows an example of the Tessellatum tile pattern. Vermiculatum is tiling method that uses fixed tile shapes with different colors as well as different angles to express directional features. Thus, Vermiculatum only permits angle variance. Figure $1 \mathrm{~b}$ shows an example of Vermiculatum tile pattern.

Palladianum: A tiling method that uses varied tile shapes with different colors, sizes and angles. By using randomly shaped tiles it can express complex features. Thus, Palladianum permits shape, size and angle variance. Figure $2 \mathrm{c}$ shows an example of the Palladianum tile pattern.

Segment based mosaic tiling: To assign the appropriate mosaic patterns to an image, we first divide image regions to three segments. According to our observations, we noticed that main features necessary to categorize traditional mosaic patterns are spatial and directional complexity. Therefore we calculated these factors in an image using complexity energy functions.

Spatial complexity: To divide the Tessellatum and Palladianum tiling patterns, we need to analyze the spatial complexity of the input image. The simplest way is to use the gradient magnitude $\mathrm{g}(\mathrm{x})$ which represents the color derivatives of an image $\mathrm{I}(\mathrm{x})$ where $\mathrm{x}$ is the coordinates $<\mathrm{x}, \mathrm{y}\rangle$. Generally, $\mathrm{g}(\mathrm{x})$ represents a spatially complex value but it only has a high value in regions with rapid color changes such as edges. Since, we need to find sufficiently smooth and large regions to contain mosaic tiles, we design the distance based complexity function $\mathrm{D}(\mathrm{x})$. Let $\mathrm{I}(\mathrm{p})$ be a complex pixel which has a minimum distance from $\mathrm{I}(\mathrm{x})$ and a higher gradient magnitude than threshold $\mathrm{e}$ (in this study, we used 0.7 as the threshold value of normalized gradient value), then we can calculate the distance based complexity function $\mathrm{D}(\mathrm{x})$ as follows:

$$
\mathrm{D}(\mathrm{x})=\mathrm{g}(\mathrm{x}) / \mathrm{x}-\mathrm{p} \mid
$$

where, $|\mathrm{x}-\mathrm{p}|$ represents a Euclidian distance between pixel $\mathrm{x}$ and $\mathrm{p}$. Distance-based energy $\mathrm{D}(\mathrm{x})$ assigns a higher

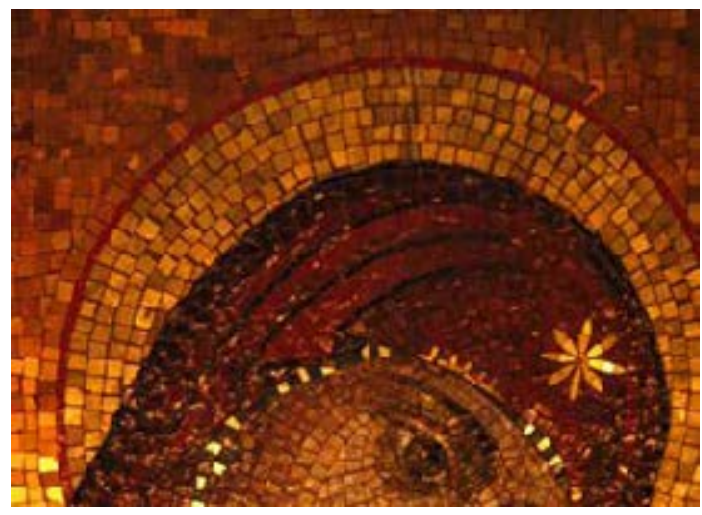

Fig. 2: Man-made mosaic art in San Giorgio Church

value for spatially complex pixels and their neighborhoods. Using $\mathrm{D}(\mathrm{x})$, we can decide if a region is complex or not which can be used as a criterion in Tessellatum and Palladianum tiling patterns. Figure 3 shows the comparison between gradient magnitude $\mathrm{g}(\mathrm{x})$ and our energy function $\mathrm{D}(\mathrm{x})$.

Directional complexity: If any region of a mosaic stylization target image has directional consistency along a specific angle, the tile should be placed to follow this direction, adhering to the Vermiculatum pattern. To calculate a value for directional consistency, we first have to calculate ETF (edge tangent flow) (Kang et al., 2009) vectors $\mathrm{F}(\mathrm{x})$ which are smoothed direction maps representing the dominant feature directions of a given image $\mathrm{I}(\mathrm{x})$. Using $\mathrm{F}(\mathrm{x})$, we designed the directional complexity function $\mathrm{C}(\mathrm{x})$ by calculating the similarity of the flow vector $\mathrm{F}(\mathrm{x})$ and its surrounding flow vector $\mathrm{F}(\mathrm{p})$ as follows:

$$
\mathrm{X}(\mathrm{x}) \frac{1}{\mathrm{~N}} \sum_{\mathrm{p} \in \Psi}|\mathrm{F}(\mathrm{x}) \cdot \mathrm{F}(\mathrm{p})|
$$

where, $\Psi$ represents a neighborhood window (in this study, we used $5 \times 5$ sized window) and $\mathrm{N}$ represents the number of pixels to normalize. Using the $C(x)$ value, we can determine that a higher $\mathrm{C}(\mathrm{x})$ value indicate that pixel $\mathrm{I}(\mathrm{x})$ has a higher directional consistency and that should be tiled using the Vermiculatum pattern.

Image segmentation and tiling: Using a two energy field $\mathrm{D}(\mathrm{x})$ and $\mathrm{C}(\mathrm{x})$, we can then divide the image into three different region types. We assume that each map is a component of a two-dimensional vector $M(x)=<D(x)$, $\mathrm{C}(\mathrm{x})>$ and then classify the vector using the k-means clustering method (Shapiroand and Stockman, 2001). To generate a smooth boundary, we applied Gaussian smoothing before the clustering. Figure 4 shows the 
(a) (b)

(c)

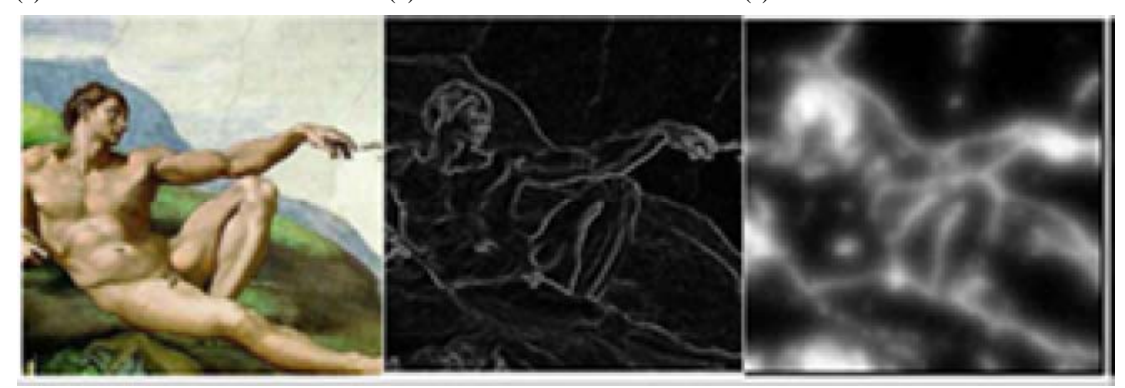

Fig. 3: Gradient energy-based distance map: a) input image; b) Gradient energy field $g(x)$ and c) Spatially complex energy using distance map $\mathrm{D}(\mathrm{x})$

(a)

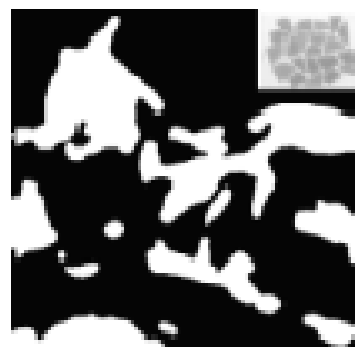

(b)

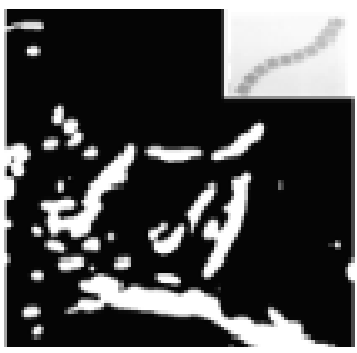

(c)

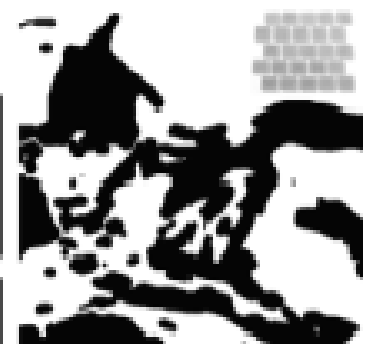

Fig. 4: a-c) Image segmentation result using spatial and directional complexity: using image Fig. 3a, three types of region is clustered by $\mathrm{k}$-means clustering algorithm

(a)

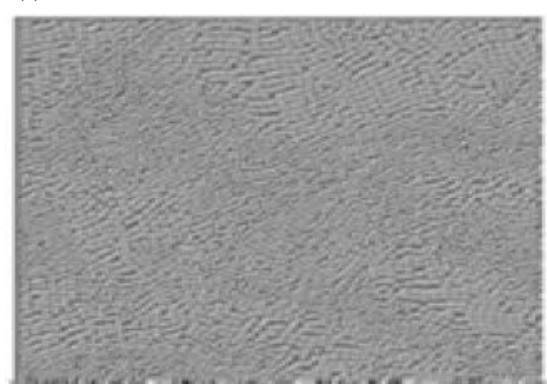

(b)

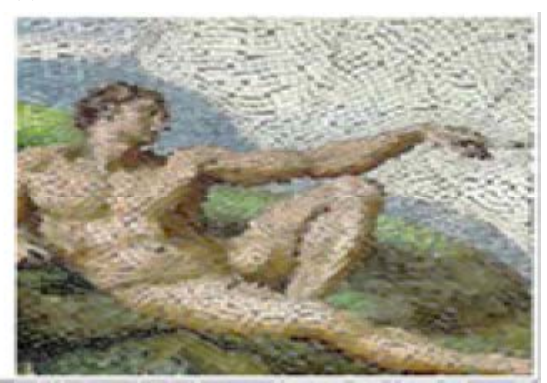

Fig. 5: Mosaic tiling using three different tile patterns: a) The shapes of tiles and b) Color assigned version of the mosaic

results of region classification and its assigned tiling patterns. Using three different regions, we place the tiles according to the assigned tile patterns. We assume that basic tiles are a rectangular have a fixed sizes and tiling order is left-to-right and top-to-bottom. For the Tessellatum region, we place the tiles at a fixed angle which minimized the gap between tiles. For the Vermiculatum region, we place the rotated tiles along the angle of the flow vector $\mathrm{F}(\mathrm{x})$ by minimizing the gap between tiles. For the Palladianum region, we randomly change the size, angle and shape of the tiles and then place the tiles by minimizing gaps. Figure 5 shows the tiling results using our classification-based tiling method.
Because our method uses three different tile patterns simultaneously, the resulting mosaic image is similar to man-made art.

Video mosaic: In this study, we extend our mosaic framework for video input. Because video is a set of image frames, we can easily apply our method to each video frame separately. However since each tile has a visually significant factor such as angle, shape and size information, differences between video frames cause serious temporal coherence problems. Motion vectors using a tracking method such as optical flow (Shapiroand and Stockman, 2001) are the conventional 

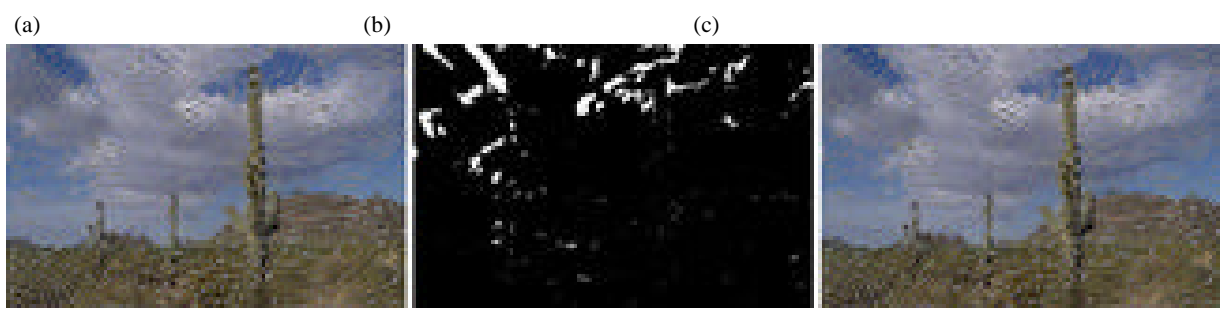

Fig. 6: Update region for video mosaic: a) Mosaic result of previous frame; b) Update region between previous frame and current frame and c) Mosaic result by retiling the up-dated region
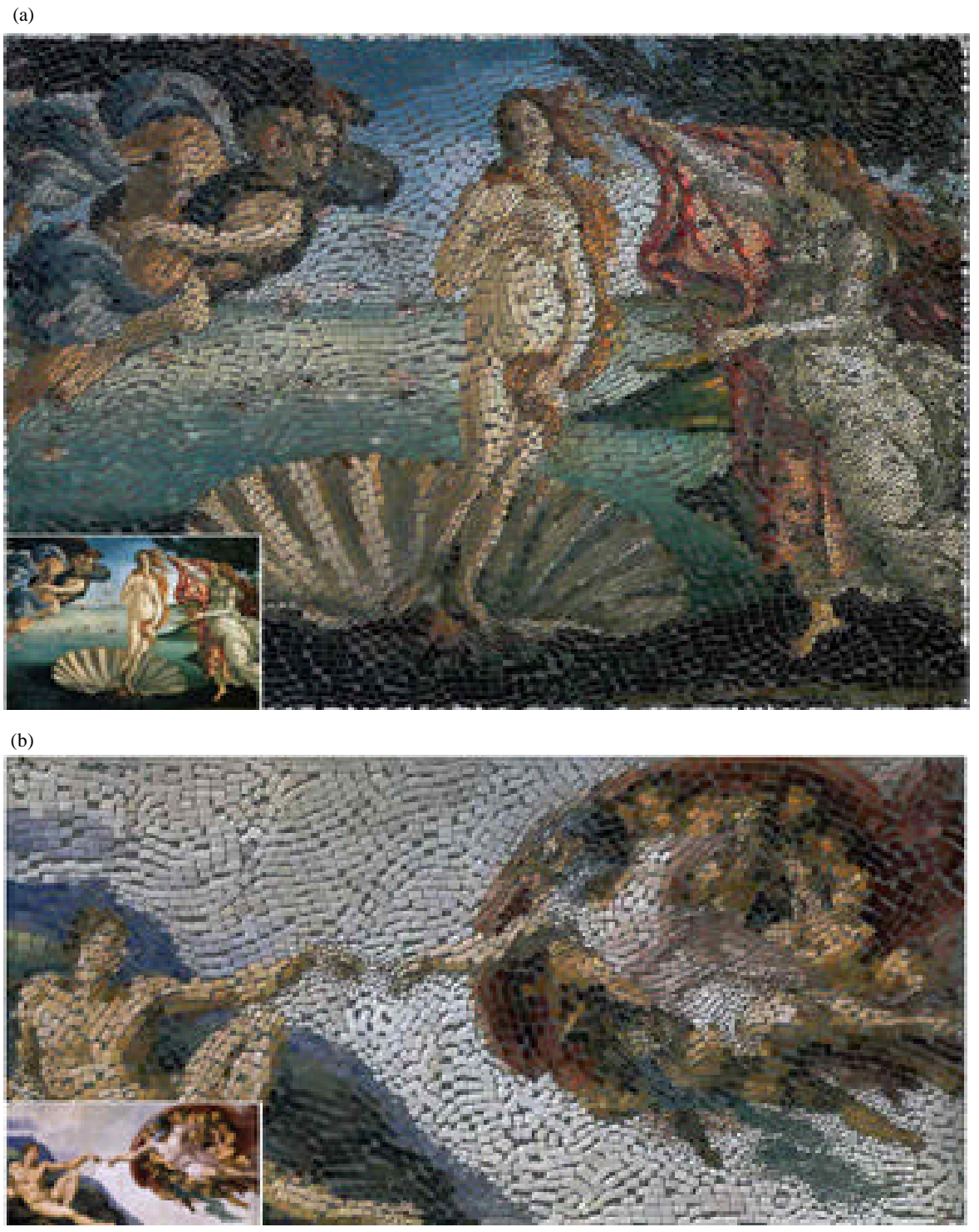

Fig. 7: a, b) Result of mosaic stylization using three traditional tiling patterns

solution to temporal coherence. However, the transformation of mosaic tiles using a motionvector may generate unwanted overlaps between tiles. To avoid overlapped artifact, tiles should be globally relocated and this cause a temporal coherence problem. Thus mosaic stylization has been limited by image application. To overcome this problem, we now present a novel approach to maintain the consistency of mosaic tiles between video frames (Fig. 6 and 7). 


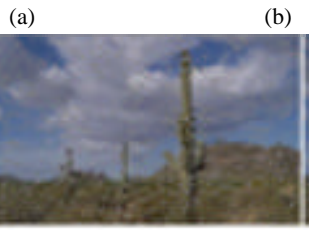

(f)

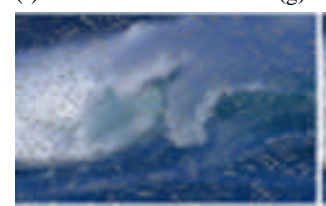

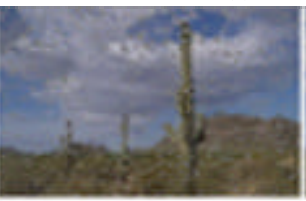

(h)

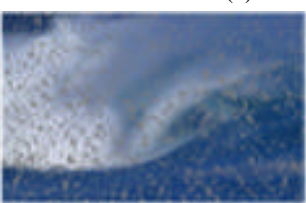

(c)

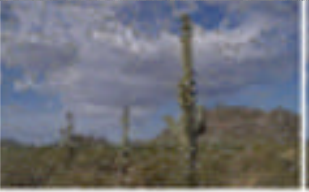

(i)

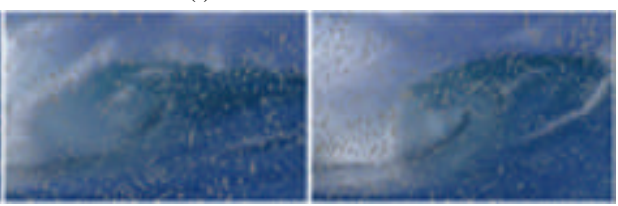

Fig. 8: a-h) Results of video mosaic stylization

(a)

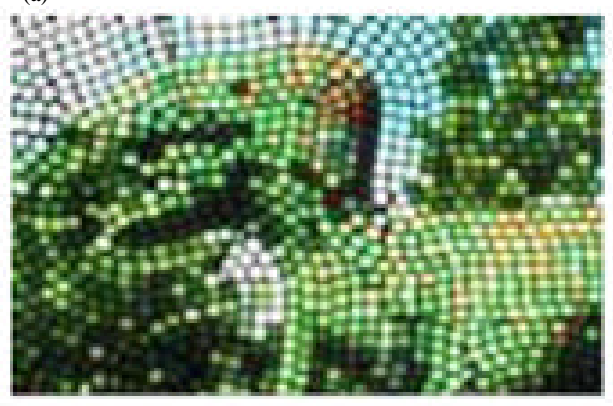

(b)

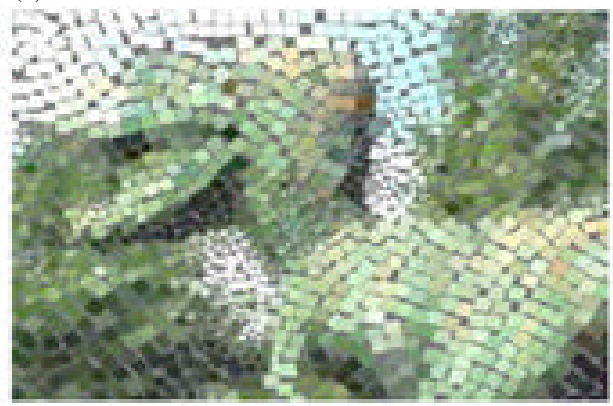

Fig. 9: Comparison of our method and previous computer generated mosaic styles: a) Elber and Wolberg's result (Elber and Wolberg, 2003 and b) Our result

(a)

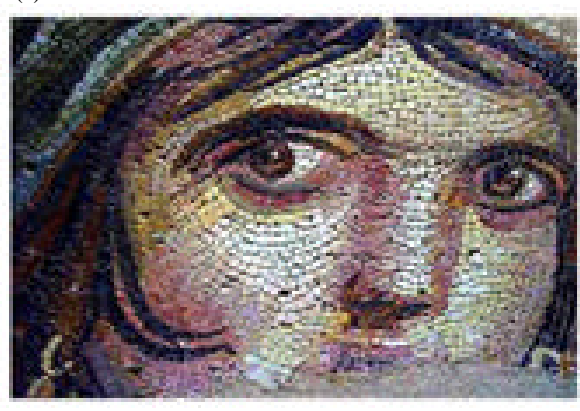

(b)

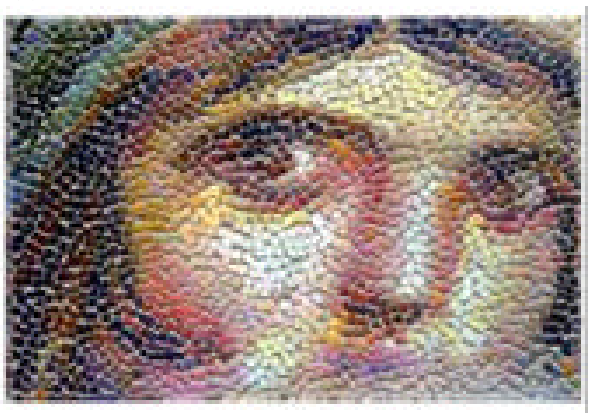

Fig. 10: Comparison of our method and manmade mosaic art: a) Man-made mosaic art and b) Our result

Our strategy is to find regions that are as small as possible and then only update the tiles in that region. Pixel differences between video frames generally represent the movement of the camera or the subject. To minimize region of difference, we first apply video stabilization method to eliminate camera movement using optical flow method. We then detect regions which represents a subject movement using the Square Summed Distance (SSD) of pixels between stabilized frames. Because noise can cause unnecessary differences between video frames, we apply image morphology (Shapiroand and Stockman, 2001) to eliminate these small regions of difference. Figure 6 shows a region requiring an update between video frames.
To consider the features of each video frame, we only replace the tiles in the region requiring an update. Because tiles on static regions have same size, shape and angle consistency, the resulting video mosaic has fewer temporal artifacts.

\section{RESULTS AND DISCUSSION}

Experimental results: Figure 7 shows the resulting mosaic image using our frame-work. Because we applied three different patterns to the segmented region, the results show visually pleasing man-made-like tiles that effectively conserve the original features of the image. Figure 8-10 shows the results of mosaic video that 
conserve the temporal coherency between frames and also effectively represent features of frame. We used a source video by Bousseau et al. (2007). To prove the advantage of our work, we compared our results with two different targets: previous computer generated mosaics and real man-made mosaics. First (Fig. 9) shows a comparison of our results with previous researcher by Elber and Wolberg. Because Elber and Wolberg concentrated on the regularity and directional consistency of mosaic tiles, the result only has artificial patterns. However, our work contains both regularity and randomness and thus effectively expresses directional features. Figure 10 shows a comparison of our result and a man-made mosaic. We used a blurred version of (Fig. 10a) as an input image and then generated a mosaic image (Fig. 10b). Although, the two results do not have equivalent tile shapes and placements, we noticed that the tile patterns are similar and this proves our system is a sufficiently similar to traditional mosaic arts.

\section{CONCLUSION}

We have presented a method to generate mosaic images that appear man-made based on our observations of traditional mosaic patterns. Using image analysis based on spatial and directional complexity, we applied adaptive tiling for natural results. We also present a novel extension of mosaic stylization using video input. While our current system only use a CPU and each image take about $10 \mathrm{sec}$ using Intel I5 Core, we expect that our algorithm would easily facilitate GPU implementation and thereby enable real-time performance in video mosaics.

It should be noted that, although, our mosaic video algorithm doses improve the temporal coherence in video mosaic results, it does not always conserve the temporal stability. When a subject's motion area between frames is rather large, temporal flickering still remains. To overcome this problem, we need to find an optimization-based approach which minimizes temporal flickering while maintaining the features of each frame (which would make it more expensive).

\section{ACKNOWLEDGEMENT}

This study is supported by 2015 Research Grant from Kangwon National University (No. 201510117).

\section{REFERENCES}

Bousseau, A., F. Neyret, J. Thollot and D. Salsin, 2007. Video watercolorization using bidirectional texture ad-vection. ACM. Trans. Graphics, 26: 104:1-104:7.

Dobashi, Y., T. Haga, H. Johan and T. Nishita, 2002. A method for creating mosaic images using Voronoi dia-grams. Proceedings of the 2002 International Conference on Eurographics Vol. 2, September 9, 2002, Eurographics Association, Europe, pp: 341-348.

Elber, G. and G. Wolberg, 2003. Rendering traditional mosaics. Visual Comput., 19: 67-78.

Kang, H., S. Lee and C.K. Chui, 2009. Flow-based image abstraction. IEEE. Trans. Visual. Comput. Graphics, 15: 62-76.

Kim, J. and F. Pellacini, 2002. Jigsaw image mosaics. Proceedings of the 29th Annual Conference on Comput-er Graphics and Interactive Techniques, July 23-26, 2002, ACM, New York, USA., pp: 657-664.

Mould, D., 2007. Stipple placement using distance in a weighted graph. Proceedings of the 3rd Eurographics Conference on Computational Aesthetics in Graphics, Visualization and Imaging, June 20-22, 2007 , Eurographics Association, Aire-la-Ville, Switzerland, ISBN: 978-3-905673-43-2, pp: 45-52.

Shapiro, L.G. and G.C. Stockman, 2001. Computer Vision. Prentice Hall, New Jersey, USA., ISBN-13: 9780130307965, Pages: 580. 